\title{
Intrapericardial Route of Administration
}

National Cancer Institute

\section{Source}

National Cancer Institute. Intrapericardial Route of Administration. NCI Thesaurus. Code C38257.

Administration of a drug within the pericardium. Intrapericardial application of therapeutic agents provides a strategy for site-specific treatment of heart or coronary diseases. The magnitude of the local advantage depends on substance exchange between the local and the blood compartments and on substance elimination from these compartments. 